\title{
TRATAMIENTOS TRADICIONALES UTILIZADOS EN UN ÁREA ENDÉMICA DE LEISHMANIASIS CUTÁNEA EN EL PERÚ
}

\author{
Roberto Pineda-Reyes ${ }^{1, a}$, Alejandro Llanos-Cuentas ${ }^{2, b}$, Mauricio Dancuart ${ }^{1, c}$
}

\begin{abstract}
RESUMEN
Con el objetivo de conocer el tratamiento utilizado por pobladores de un área endémica de leishmaniasis cutánea (LC) previo a su atención en un servicio de salud, se realizó un estudio de corte transversal en un poblado de Lima, Perú. Se censó a toda la población y se recolectaron datos demográficos y antecedentes de LC. De los 254 habitantes censados, 41,7\% (106/254) refirieron haber tenido LC y de ellos solo la mitad acudieron a un centro de salud. 76/106 $(71,7 \%)$ usaron algún tratamiento tradicional como primera medida y solo $23,6 \%(25 / 106)$ acudieron a un centro de salud sin manipular sus lesiones. Un alto porcentaje de personas potencialmente infectadas por LC manipulan y tratan sus lesiones con métodos tradicionales antes de acudir a un centro de salud, lo que podría interferir con el diagnóstico y efectividad del programa de tratamiento implementado por el Ministerio de Salud.
\end{abstract}

Palabras clave: Leishmaniasis cutánea; Tratamiento; Medicina tradicional (fuente: DeCS BIREME).

\section{TRADITIONAL TREATMENTS IN AN ENDEMIC AREA OF AMERICAN CUTANEOUS LEISHMANIASIS IN PERU}

\begin{abstract}
In order to know the first-choice treatment by villagers of an endemic area of Cutaneous Leishmaniasis (CL) prior to medical attention in a health care center, a cross sectional study was realized in Pichupampa town. A census was made in order to collect demographic data and previous history of CL. 254 participants were surveyed. $41.7 \%(106 / 254)$ of the village had $C L$ at least once in their lives and only half of them went to a health center to seek for primary care. 76/106 $(71.7 \%)$ used some traditional treatment as their first choice and only $23.6 \%(25 / 106)$ subjects went to a health-care center without manipulation of their lesions. It's evident that a high percentage $(71.7 \%)$ of people potentially infected by CL manipulate and treat their lesions with traditional treatments prior to professional health-care, actions that could interfere with the diagnosis and effectiveness of the program implemented by the Health Ministry.
\end{abstract}

Key words: Leishmaniasis, cutaneous; Treatment; Medicine, traditional (source: MeSH NLM).

\section{INTRODUCCIÓN}

La leishmaniasis cutánea (LC) es una enfermedad producida por un parásito del género Leishmania, considerada dentro de las enfermedades más olvidadas del mundo, afecta a poblaciones que viven en áreas rurales, la mayoría en situaciones de pobreza y pobreza extrema ${ }^{(1,2)}$. Según la OMS (2010), la prevalencia se encuentra en aumento: 350 millones de personas en el mundo se encuentran en riesgo de contraer leishmaniasis y se reportan aproximadamente 2 millones de casos nuevos al año ${ }^{(2)}$.
En el Perú, la leishmaniasis es la segunda endemia de tipo tropical y la tercera causa de morbilidad por enfermedades infecciosas luego de malaria y tuberculosis (1). Cerca del $74 \%$ del área del Perú es considerada endémica, abarcando los valles interandinos y la sierra, así como la selva alta y selva baja ${ }^{(1,3)}$. Producto de esto, las poblaciones expuestas han desarrollado una cultura de tratamientos tradicionales, es decir, aplicación de medios físicos y/o químicos en base a las creencias y a experiencias transmitidas de generación en generación.

Facultad de Medicina, Universidad Peruana Cayetano Heredia. Lima, Perú.

Instituto de Medicina Tropical Alexander von Humboldt, Universidad Peruana Cayetano Heredia. Lima, Perú.

Médico cirujano; ${ }^{\mathrm{b}}$ médico especialista en Enfermedades Infecciosas y Tropicales, $\mathrm{PhD} ;{ }^{\mathrm{c}}$ estudiante de Medicina

Recibido: 09-04-15 Aprobado: 19-08-15 
La manipulación de las lesiones usando tratamientos tradicionales antes de la evaluación por el personal de salud, constituye un problema de salud pública importante, debido a que existe aumento de inflamación en la zona de la herida, se produce necrosis y las características típicas de las lesiones se alteran, pudiendo incluso disminuir la sensibilidad del diagnóstico parasitológico ${ }^{(3-7)}$. Estos factores afectan la efectividad de los programas de diagnóstico y control.

El objetivo del presente estudio fue conocer la frecuencia y tipo de tratamiento que usan los pobladores que viven en áreas endémicas de LC como primera medida previa a la atención en un servicio de salud.

\section{EL ESTUDIO}

Se realizó un estudio poblacional de corte transversal en el poblado de Pichupampa, situado a 2250 metros de altitud, ubicado en el distrito de Leoncio Prado, provincia de Huara, departamento de Lima, en agosto del año 2012. Lima se encuentra entre los departamentos que reportan más casos de LC, siendo Leoncio Prado el distrito que más casos reporta junto con el distrito de Ámbar $(27,5 \%$ y $29 \%$ respectivamente) ${ }^{(8,9)}$. Leoncio Prado consta de ocho principales poblados: Santa Cruz, Huamboy, Huanangui, Parán, Pichupampa, Santo Domingo de Apache, Tantán y Huampán, de los cuales Pichupampa, Santo Domingo de Apache y Parán son los más grandes, contando con poblaciones que bordean los 300 habitantes.

Previa autorización del presidente de la comunidad y su comité directivo, se elaboró un mapa de la comunidad, ubicando las casas. En cada casa se pidió autorización para realizar el censo, lo cual fue aceptado por el $100 \%$ de los jefes de familia; se visitó el total de casas. En el censo, además de las variables demográficas, se incluyó el antecedente de LC en algún miembro de la familia. Si este dato era positivo, se procedió a invitar al jefe de la familia a participar, explicando la razón y el motivo del estudio. A aquellos que aceptaron se les explicó el consentimiento informado y se les entregó el documento para que lo leyeran y firmaran.

Luego, se ubicó a la persona que tomaba las decisiones sobre problemas de salud de la familia, quien fue la que respondió el cuestionario. Sin embargo, durante la entrevista, usualmente, estuvieron presentes los otros miembros de la familia, quienes también opinaron sobre las preguntas del cuestionario. Al momento de seleccionar a la persona que iba a responder la encuesta se tomó como criterio que tenga 18 años o más, y excluyó a los participantes con residencia transitoria (menor a 6 meses) en la comunidad o con limitaciones intelectuales para responder adecuadamente el cuestionario.
También se examinó a la(s) persona(s) con antecedentes de LC en la familia y se verificó el diagnóstico clínico identificando la cicatriz característica, o si tenía una lesión activa.

La definición de Medicina Tradicional fue tomada de las Pautas Generales para las Metodologías de Investigación y Evaluación de la Medicina Tradicional, misma definición que usa la OMS: "Todo el conjunto de conocimientos, aptitudes y prácticas basados en teorías, creencias y experiencias indígenas de las diferentes culturas, sean o no explicables, usados para el mantenimiento de la salud, así como para la prevención, el diagnóstico, la mejora o el tratamiento de enfermedades físicas o mentales ${ }^{(10) . "}$

El análisis estadístico se realizó en el programa STATA/SE 12.0 para Windows. Se utilizó estadística descriptiva; se determinaron frecuencias absolutas y relativas para las variables categóricas como: sexo (masculino/femenino), y LC (presente/ausente). Para variables cuantitativas como edad se usó la media aritmética. Se utilizó la prueba de chi cuadrado para determinar asociación entre las variables categóricas: sexo masculino o femenino y presencia o no de LC. Se consideró significativo un valor de $p<0,05$.

El estudio fue aprobado por el Comité de Ética Institucional del Hospital Nacional Cayetano Heredia.

\section{HALLAZGOS}

Las características generales de la población se presentan en la Tabla 1. La edad promedio fue $25,89 \pm 18,88$ años. $55,1 \%$ fueron varones. $1,9 \%$ fueron analfabetos, $21,3 \%$ con secundaria completa y $2,8 \%$ educación superior. $33,9 \%$ del total se dedicaban a la agricultura y el mismo porcentaje se registró para estudiantes. El $20,5 \%$ se dedicaban a quehaceres domésticos.

Se seleccionaron a todos los habitantes de la comunidad que se encontraban presentes al momento de la incursión, que fueron un total de 254 participantes entre adultos y niños. Se censaron 61 (79,2\%) del total de casas (77) de la comunidad. El resto de casas no se registraron debido a que las familias estaban ausentes ya sea por trabajo, viaje u otro factor externo. $81,8 \%$ de las familias censadas tuvieron al menos una persona con antecedente de LC.

De los 254 participantes, 106 (41,7\%) refirieron haber tenido LC alguna vez en su vida. De este grupo, 71/106 $(66,9 \%)$ refirieron haber recibido alguna vez tratamiento con antimonio pentavalente; 36/106 (33,9\%) refirieron 
Tabla 1. Características de los participantes

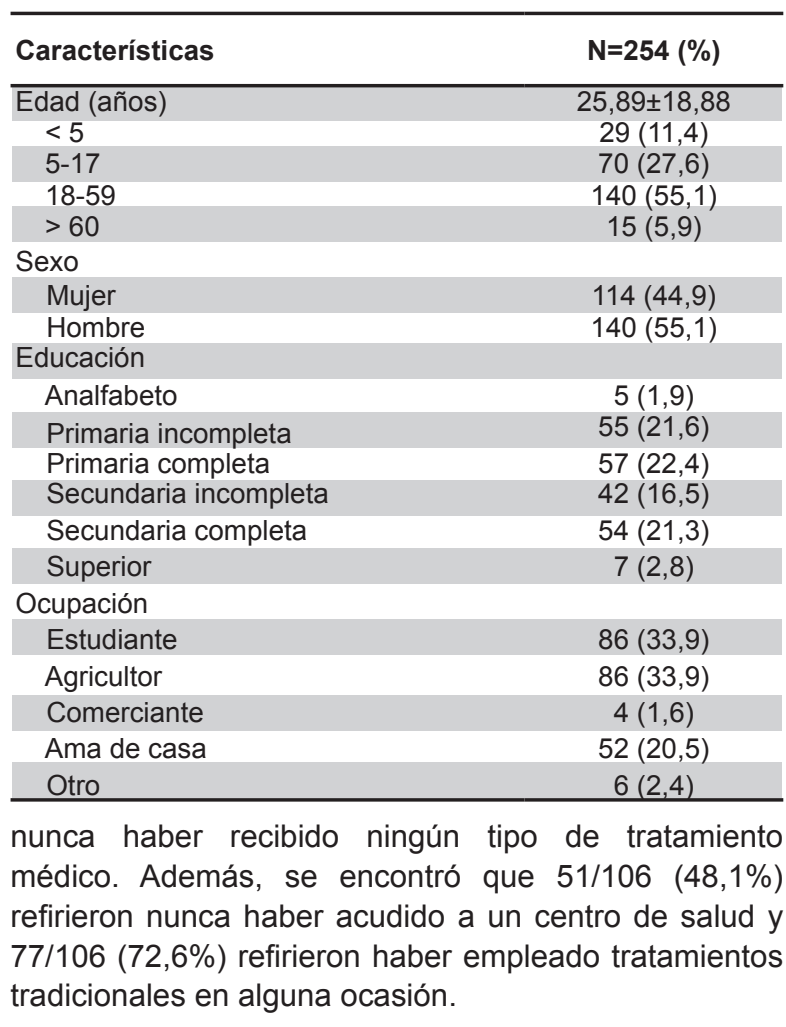

Sobre la primera medida de elección ante la aparición de una lesión sospechosa de LC, 76/106 (71,7\%) usaron tratamientos tradicionales, 25/106 (23,6\%) acudió a un centro de salud y en $4 / 106(3,7 \%)$ casos hubo autotratamiento con antimoniales (Figura 1); 31/106 $(29,2 \%)$ acudieron a un centro de salud como segunda conducta ante la aparición de una lesión sospechosa.

De las 76 personas que emplearon tratamientos tradicionales como primera medida, solamente 20 $(26,3 \%)$ refirieron haberse curado con estos medios, $3 / 76(3,9 \%)$ empeoraron y $40 / 76(52,6 \%)$ no curaron y tuvieron que buscar una segunda opción de tratamiento, la cual fue autotratamiento con antimoniales en 12 personas y en 28 personas acudir a un centro de salud. Solo una persona tenía una lesión activa al momento de la investigación (con diagnóstico y tratamiento por el centro de salud). En 11/76 casos no se recordó si la herida curó con tratamientos tradicionales o si remitió espontáneamente y en 1 caso la herida no curó con tratamientos tradicionales pero luego remitió sin necesidad de tratamiento médico.

Acerca de los tipos de tratamientos tradicionales, 47 $(44,3 \%)$ pobladores refirieron emplear hierbas, hojas y otras plantas que crecen en los alrededores, la categoría que le sigue en frecuencia fue la aplicación de ácidos, como ácido cítrico, ácido de batería, de pila, entre otros (38 personas: $35,8 \%$ ); luego se encuentra la aplicación de químicos como alcohol, mentol, etc. (16 personas: $15,1 \%$ ) y por último la aplicación local de antibióticos automedicados (5 personas: $4,7 \%$ ). Se

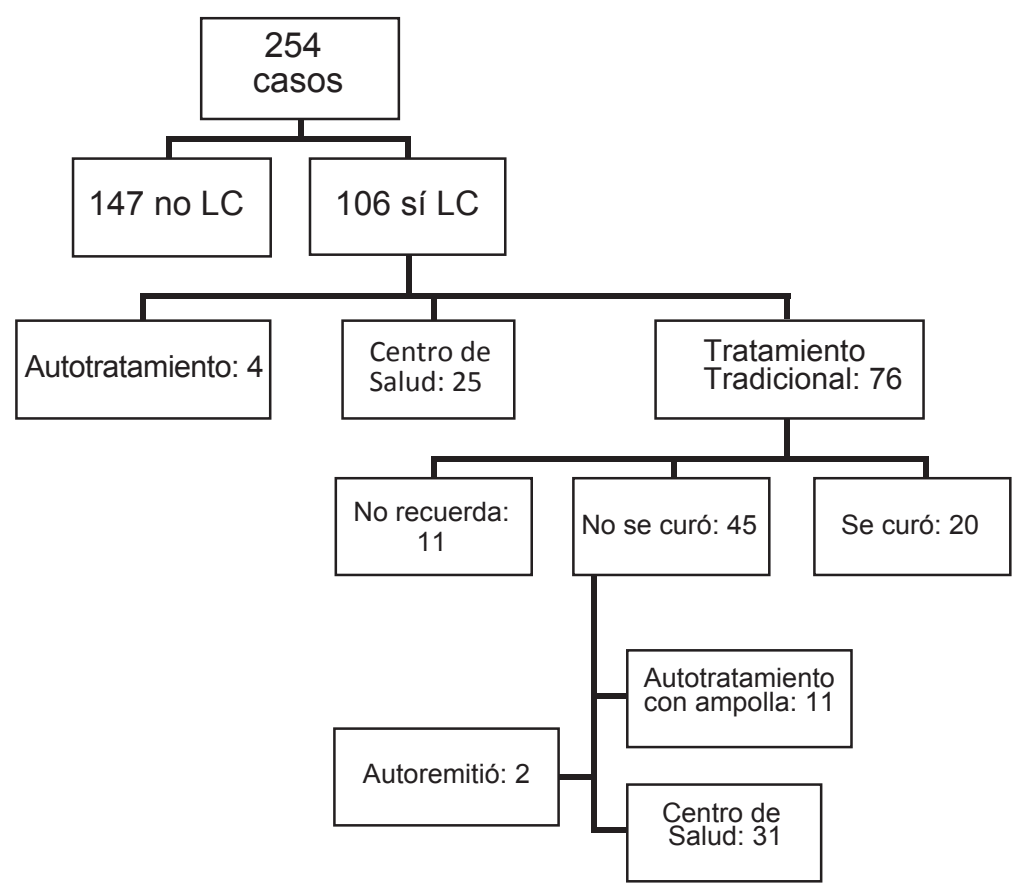

Figura 1. Algoritmo de casos de leishmaniasis cutánea (LC) en el poblado de Pichupampa. 
Tabla 2. Tratamientos tradicionales empleados por los pobladores de Pichupampa

\begin{tabular}{|c|c|c|c|}
\hline $\begin{array}{l}\text { Tratamiento } \\
\text { tradicional }\end{array}$ & Nombre científico & $N=106$ & $(\%)$ \\
\hline Limón con sal & & 25 & $(23,6)$ \\
\hline "Leche-lechera" & Euphorbia heterophylla & 21 & $(19,8)$ \\
\hline Ácido de batería & & 8 & $(7,5)$ \\
\hline "Oropimente" & Sedum telephium & 7 & $(6,6)$ \\
\hline Alcohol & Etanol & 7 & $(6,6)$ \\
\hline Llantén & Plantago major & 5 & $(4,7)$ \\
\hline Limón & Citrus limon & 5 & $(4,7)$ \\
\hline Hierba "tara" & Caesalpinia spinosa & 4 & $(3,8)$ \\
\hline Mentol & $\begin{array}{l}\text { 5-metil-2-isopropil } \\
\text { ciclohexa-1-ol }\end{array}$ & 4 & $(3,8)$ \\
\hline Hierba "chupa sangre" & Oenothera rosea & 3 & $(2,8)$ \\
\hline Ajo & Allium sativum & 3 & $(2,8)$ \\
\hline Ortiga & Urtica dioica $L$. & 2 & $(1,9)$ \\
\hline Vick Vaporud $^{\circledR}$ & & 2 & $(1,9)$ \\
\hline Ampicilina & & 2 & $(1,9)$ \\
\hline Hierba "San Carlos" & & 1 & $(0,9)$ \\
\hline Otras hierbas & & 1 & $(0,9)$ \\
\hline Sulfanil ${ }^{\circledR}$ & & 1 & $(0,9)$ \\
\hline Terramicina & & 1 & $(0,9)$ \\
\hline "Yeso" & & 1 & $(0,9)$ \\
\hline "Penicilina en polvo" & & 1 & $(0,9)$ \\
\hline Timolina & & 1 & $(0,9)$ \\
\hline Sal & Cloruro de sodio & 1 & $(0,9)$ \\
\hline
\end{tabular}

encontró, además, que los tratamientos tradicionales más populares son la aplicación de "limón con sal" y de la hierba "leche-lechera" (Euphorbia heterophylla), luego se encuentran el uso de desinfectantes como alcohol y quemar la herida con "ácido de batería", etc. (Tabla 2).

\section{DISCUSIÓN}

Pichupampa es una población con alta prevalencia de LC $(41,7 \%)$, encontrándose que en el $81,8 \%$ de las familias censadas por lo menos un integrante tenía historia de LC. Cabe resaltar que esta cifra podría estar subestimada por las migraciones, así algunas familias que viven actualmente en esta localidad vienen de lugares donde la LC no es endémica, mientras que los miembros jóvenes de familias que llevan viviendo en Pichupampa más de tres generaciones han migrado a ciudades cercanas como Huacho.

En esta comunidad, según señalan sus pobladores más antiguos, "siempre ha habido uta" por lo que existe una cultura popular para el diagnóstico y tratamiento de esta enfermedad que data de más de cincuenta años. Los pobladores de la zona suelen reconocer la lesión característica de LC pero su problema es el tratamiento.

El $71,7 \%$ de los participantes con antecedente de LC se automedicaron utilizando tratamientos tradicionales de distintos tipos como su primera opción de tratamiento, lo cual, en la práctica, es aplicación de sustancias cáusticas que queman la lesión. En contraste, solo el $23,6 \%$ eligen acudir a un servicio de salud como su primera opción de tratamiento; se observó que este grupo estuvo integrado por familias más jóvenes, lo cual sugiere un cambio de actitud en la comunidad; sin embargo, aún la mayoría de los pobladores prefieren utilizar el tratamiento tradicional antes que el tratamiento médico.

La prevalencia acumulada de LC que se halló fue menor en comparación a datos de estudios anteriores en áreas endémicas en 1987-1988 ${ }^{(3,11)}$, en los cuales se evidenciaba que alrededor de $70-80 \%$ de los pobladores tenían cicatrices características de la enfermedad; sin embargo, fue similar a estudios en zonas endémicas de otros países de Sudamérica como Ecuador en 1994 y 2001: $47 \%{ }^{(6)}$ y 49,7\% ${ }^{(12)}$ respectivamente y en Colombia en 1991: $29,9 \%$ (7); esto podría deberse a múltiples factores como mejoras en el programa de control de la enfermedad, mejor educación y conocimiento de los pobladores, migración a la ciudad, etc.

Es importante mencionar que si bien Pichupampa es un área endémica en LC, los resultados de este estudio están basados en una encuesta transversal y la visualización de la cicatriz característica de la enfermedad, pero sin confirmación por estudios de laboratorio. Esto establece un sesgo ya que otras enfermedades dermatológicas como esporotricosis podrían presentar lesiones con características similares. Por otra parte, según se demostró en estudios anteriores realizados por LlanosCuentas y Davies ${ }^{(11)}$, en áreas endémicas la lesión clásica de LC correlaciona adecuadamente con la positividad en el test de Montenegro.

No se encontraron diferencias de la prevalencia en cuanto a sexo, a pesar de que en la comunidad los varones predominaban en número, los casos de LC en varones no superaron significativamente al grupo de las mujeres $(p=0,687)$, lo cual es una característica de la LC andina cuya transmisión es peridomiciliaria, a diferencia de la leishmaniasis selvática que es epidemiológicamente más predominante en varones y que tiene una transmisión ocupacional.

Se observó, además, que la mayoría de veces el varón es el jefe de familia, sin embargo, la persona que toma la decisión del manejo ante la enfermedad suele ser la mujer (madre de familia). Estos hallazgos hacen notar con claridad a quiénes debe ir dirigida la intervención educativa. Por otra parte, casi la mitad $(48,1 \%)$ de las personas con historia de LC refirieron nunca haber acudido a un Centro de Salud, sin embargo 71/106 (66,9\%) recibieron alguna vez tratamiento con antimoniales, es decir, los pobladores consiguen las ampollas por sus propios medios o hay terceras personas que se las administran sin supervisión de un centro de salud (Figura 1).

En cuanto al tipo de tratamiento empleado, los resultados coinciden con estudios realizados en Ecuador y Colombia 
mencionados anteriormente, en los cuales lo más frecuente es la aplicación tópica de plantas: hierbas, hojas, flores, etc., seguida por la terapia de quemadura con productos químicos, ácidos y aplicación de sustancias calientes. Se encontró que la costumbre más popular es la aplicación de limón con sal, la cual se realizó en la tercera parte de las personas que recibieron tratamientos tradicionales. La segunda costumbre más frecuente fue el uso de la hierba "leche-lechera" (Euphorbia heterophylla) de manera local en la herida ( $19,8 \%$ de casos), esta es una planta que crece en los alrededores de la comunidad, en el monte, cuya fama acerca de sus beneficios curativos se ha transmitido de generación en generación, entre las familias más antiguas de Pichupampa y algunas otras comunidades aledañas como Santo Domingo de Apache, sin embargo, su actividad leishmanicida ha sido catalogada como moderada a pobre ${ }^{(13)}$

Otro hallazgo es que en más de la mitad de los casos en los que se emplearon tratamientos tradicionales como primera opción, la lesión no se curó e incluso hasta empeoró (tasa de curación usando tratamientos tradicionales: $26,3 \%$ ), lo cual cambia las características de la lesión primaria y así se podría alterar la carga parasitaria de la herida y el correcto diagnóstico parasitológico, así como, potencialmente, incrementar el tiempo de curación.

En conclusión, es evidente que un alto porcentaje de personas potencialmente infectadas con LC manipulan sus lesiones usando tratamientos tradicionales antes de acudir a un centro de salud, esto podría disminuir la positividad del frotis y del cultivo in vitro en pacientes con lesiones manipuladas, sin embargo, aún se desconoce el impacto del uso de los tratamientos tradicionales en el diagnóstico de LC.

Contribuciones de autoría: la concepción y el diseño del artículo fue por ALLC. REPR y JMDM participaron en la recolección y obtención de resultados. REPR y ALLC realizaron el análisis e interpretación de datos, la redacción del artículo y la aprobación de la versión final.

Fuentes de financiamiento: Unidad de Leishmania y Malaria, Instituto de Medicina Tropical Alexander von Humboldt, Universidad Peruana Cayetano Heredia.

Conflictos de interés: ninguno.

\section{REFERENCIAS BIBLIOGRÁFICAS}

1. Perú, Ministerio de Salud, Oficina General de Epidemiología. Leishmaniasis. Módulos Técnicos. Serie Documentos Monográficos Nº. Lima: MINSA; 2000.

2. World Health Organization. Control of the leishmaniasis. Technical report series 949. Geneva: WHO; 22-26 March 2010.

3. Guerra H. Distribution of Leishmaniasis in Peru. En: Walton BC, Wijeyaratne PM, Modabber F, ed. Research on Control Strategies for the Leishmaniases: Proceedings of an International Workshop held in Ottawa, Canada, 1-4 June 1987. Manuscript Report 184e. Otawa: IDRC; 1988. p. 135-47.

4. Llanos-Cuentas A, Chang OJ, Echevarria J. Adapting leishmaniasis treatment to peripheral health centers and communities: adapting anti-leishmanial therapy to peripheral health services and community health workers. En: Leishmaniasis Control Strategies: A critical evaluation of IDRCsupported Research. IDRC, Ottawa: IDRC; 1992. p. 325-332.

5. Vázquez ML, Kroeger A, Lipowsky R, Alzate A. Conceptos populares sobre la leishmaniasis cutánea en Colombia y su aplicabilidad en programas de control. Bol Oficina Sanit Panam. 1991 May;110(5):402-12.

6. Weigel MM, Armijos RX, Racines RJ, Zurita C, Izurieta R, Herrera E, et al. La leishmaniasiscutáneaen la región subtropical del Ecuador: percepciones, conocimientos y tratamientos populares1. Bull Pan Am Health Organ. 1994 Jun;28(2):142-55.

7. Isaza DM, Restrepo BN, Arboleda M, Casas E, Hinestroza H, Yurgaqui T. La leishmaniasis: conocimientos y prácticas en poblaciones de la costa del Pacífico de Colombia. Rev Panam Salud Publica. 1999 Set;6(3):177-84.

8. Perú, Ministerio de Salud. Dirección General de Epidemiología. Situación epidemiológica de la Leishmaniosis en el Perú, 2013 (SE 52). Bol Epidemiol. 2014;23801):6-10.

9. Perú, Ministerio de Salud. Análisis de la Situación de Salud Región Lima 2011. Lima: Dirección Regional de Salud Lima; 2011.

10. World Health Organization. General Guidelines for Methodologies on Research and Evaluation of Traditional Medicine. Geneva: WHO; 2000.

11. Llanos-Cuentas A, Davies C. Epidemiological studies on Andean
Cutaneous Leishmaniasis and their significance for designing a control strategy. En: Leishmaniasis Control Strategies: A critical evaluation of IDRCsupported Research. IDRC, Ottawa: IDRC; 1992. p. 286-302.

12. Weigel MM, Armijos RX. The traditional and conventional medical treatment of cutaneous leishmaniasis in rural Ecuador. Rev Panam Salud Publica. 2001 Dec;10(6):395-404.

13. Valadeau C, Pabon A, Deharo E, Albán-Castillo J, Estevez Y, Lores FA, et al. Medicinal plants from the Yanesha (Peru): Evaluation of the leishmanicidal and antimalarial activity of selected extracts. J Ethnopharmacol. 2009 Jun 25;123(3):413-22.

14. Perú, Ministerio de Salud. Norma técnica: Diagnóstico y tratamiento de Leishmaniosis en el Perú. Lima: MINSA; 2005.

Correspondencia: Roberto Enzo Pineda Reyes Dirección: Calle Bayóvar Sur 121

Urbanización Prolongación Benavides

Santiago de Surco

Teléfono: 995112164

Correoelectrónico: roberto.pineda.r@upch.pe 